\title{
Management of Labour
}

\section{Management of normal labour}

\section{Introduction}

The aim of this guideline is to provide recommendations to care providers in the management of a healthy woman with a single fetus in labour at term (37-42weeks). It does not cover the care of women with complicated pregnancies.

The objective of this guideline is to ensure optimal management of women in labour, detect any abnormality, take appropriate action, prevent complications and consequently make childbirth safer; and also to make sure that these women are treated with respect and compassion, kept well informed and well supported throughout labour

\section{Diagnosis of labour}

Labour is diagnosed by the presence of regular, painful intermittent contractions, which are of increasing frequency, duration and intensity, leading to progressive cervical effacement and dilatation.

Note: for the purpose of this guideline, labour is also diagnosed in the presence of painful contractions occurring at a frequency of 2 in 10 minutes or more.

\section{Definitions:}

Latent phase of the first stage of labour - from the commencement of labour to a cervical dilatation of up to $4 \mathrm{~cm}$. (This is a period of time, not necessarily continuous, when there are painful contractions and some cervical changes including cervical effacement and dilatation up to $4 \mathrm{~cm}$ take place)

Active phase of the first stage of labour commences at a cervical dilatation of $4 \mathrm{~cm}$ and ends with full dilatation. (There are regular painful contractions and progressive cervical dilatation from $4 \mathrm{~cm}$ up to full dilatation)

If the diagnosis of labour is uncertain, observation should continue and reassessment made in four hours.

Any woman who is diagnosed as not being in labour, but continues to complain of pain, would require careful reassessment by an experienced medical officer. Possible diagnoses of placental abruption and non-obstetric causes should be considered. Fetal compromise should be excluded.

\section{Management of labour}

\subsection{General considerations}

3.1.1. Communication between women and healthcare professionals/workers

- Greet the mother with a smile and a personal welcome

- Treat her with respect and dignity

- Assure privacy

- Establish a good rapport with the labouring woman asking her about her wants and concerns and address them

- Maintain a calm and confident approach which will reassure women that the situation is under control

- Assess the woman's knowledge of strategies for coping with pain and provide balanced information to find out which available approaches are acceptable to her

- Ask her permission before all procedures and observations, focusing on the woman rather than technology or the documentation

\subsubsection{Preparation of mothers to transfer to labour room}

- Shaving or trimming of perineal hair may be necessary to facilitate unhindered performance and repair of the episiotomy.

- Efforts must be made to minimize faecal soiling. Where an enema is deemed necessary, a medicated enema is recommended.

(These two steps should not be considered mandatory)

- Women should be encouraged to have a companion of her choice during labour, depending on the facilities and clinical situation.

\subsubsection{Documentation}

- Admit the mother to the labour room and complete the 'handing over' form.

- Keep relevant notes on the BHT and start a partograph.

- Review clinical notes and reassess risk factors.

- Accurate documentation of all observations and interventions must be made, with timing.

- All obstetric examinations and procedures carried out must be documented in the clinical notes. Each entry must be accompanied by a plan for management and be signed by the responsible person. 


\subsubsection{Mobilization and positioning}

- Women should be encouraged and helped to move about and adopt whatever positions they find most comfortable throughout labour.

- They need to be encouraged to void urine regularly.

\subsubsection{Eating and drinking in labour}

- Mothers must be encouraged to consume clear, non-fizzy liquids during labour. Isotonic solutions such as oral rehydration fluid and coconut water are more beneficial than water.

- In addition to clear fluids, women in the latent phase may consume light solids e.g. biscuits and fruits.

\subsubsection{Hygiene during labour}

- Strict asepsis must be maintained during labour.

- Instruments should be available in packets.

- Use proper hand washing technique.

- Use of double gloves and disposable gloves is encouraged.

\subsubsection{Pain relief in labour}

Relief of pain should be a major consideration (please refer guideline on pain relief during labour).

\subsection{Management of the three stages of labour}

The practice of maintaining a labour, room 'notice board' - a 'white board' in which the status of all women in labour is summarized and updated regularly is encouraged. This would convey at a glance to all care providers women who require additional attention. The age, parity status, risk factors, salient findings at each assessment and any abnormalities noted must be included in this.

\subsubsection{Management of the first stage of labour}

\subsubsection{Latent phase}

It is important to recognize the latent phase of labour, since its prolongation could lead to maternal exhaustion, dehydration and acidosis, leading to fetal compromise and dysfunctional labour.

Women in the latent phase of labour would be best managed in the antenatal ward.

Women in the latent phase of labour must be assessed on a regular basis, as follows:
- Check the fetal heart and maternal pulse half hourly;

- Check temperature four hourly;

- Consider vaginal examination four hourly, depending on the contraction pattern and initial cervical dilatation;

- Document the colour of amniotic fluid if the membranes rupture;

- Use of a sanitary pad may indicate early the presence of meconium.

- Consider the requirement for analgesia.

It is important to inform the mother and reassure her that it is common to have slow progress in the latent phase.

The latent phase is considered prolonged when it lasts more than 12 hours in a primigravida and 8 hours in a multigravida. In these situations an experienced medical officer (with a minimum one year of experience in the field) must reassess the mother with a view to augmentation of labour.

\subsubsection{Active phase}

\subsubsection{2a. Admitting women to the labour room}

All pregnant women diagnosed as being in active phase of the first stage of labour need to be admitted to the labour room.

The initial assessment of a woman at the labour room should include:

- Listening to her story, considering her emotional and psychological needs and reviewing her clinical records

- Physical observation: temperature, pulse, blood pressure

- Length, strength and frequency of contractions

- Abdominal palpation: fundal height, lie, presentation, position and station

- Vaginal loss: show, liquor, blood

- Assessment of woman's pain including her wishes for coping with labour along with the range of options for pain relief

- The fetal heart rate (FHR) should be auscultated preferably with a hand held Doppler for a minimum of 1 minute immediately after a contraction

- The maternal pulse should be recorded to differentiate between maternal pulse and FHR

- A vaginal examination should be offered

Health care professionals who conduct vaginal examination should:

- Be sure that there is a valid indication for vaginal examination that it will add important information to the decision making process 
- Be aware that for many women who may already in pain, highly anxious and in an unfamiliar environment, vaginal examination can be very distressing

- Ensure the woman's consent, privacy, dignity and comfort

- Explain the reason for examination and what will be involved, and

- Explain the findings and their impact sensitively to the woman

\subsubsection{2b. Management of active phase of first stage}

Monitoring must be conducted as instructed in the partogram and findings recorded accordingly.

Use of a sanitary pad may indicate early presence of meconium.

Women in the active phase of labour must be assessed on a regular basis, as follows:

- Check the fetal heart and maternal pulse every 15 minutes;

- Check temperature and blood pressure four hourly;

- Vaginal examination four hourly or earlier, depending on the clinical situation;

- Frequency of contractions should be monitored as follows:

The interval between two contractions should be assessed by palpation of the abdomen. During active labor usually there are at least three contractions per ten minutes. In other words the interval between two contractions should be three minutes

- Document the colour of amniotic fluid if the membranes rupture;

- Consider the requirement for analgesia, (which now becomes more important).

Intermittent auscultation of the fetal heart is best performed using hand-held Doppler devices. The fetal heart rate must be counted for one minute beginning immediately after a contraction.

The mother may continue to consume clear fluids in the active phase.

She must be encouraged to assume any position that she is comfortable in and to avoid the dorsal position.

Women who have the following conditions are recommended to be have to continuous electronic fetal monitoring:
- Significant meconium staining of amniotic fluid,

- Abnormal fetal heart rate detected by intermittent auscultation ( $<110$ beats per minute; $>160$ beats per minute; any decelerations after a contraction)

- Fresh vaginal bleeding and

- Maternal pyrexia

In women with spontaneous labour progressing normally; routine early amniotomy and, use of oxytocin is not recommended.

\subsubsection{Delayed progress of first stage of labour}

Delayed progress is diagnosed when there is progress of less than two $\mathrm{cm}$ in four hours.

Slowing of progress in a woman who has previously been progressing satisfactorily must also be considered as a delay.

It is extremely important that delay in progress is assessed by an experienced medical officer. This assessment must take into account:

- the uterine contractions,

- descent and position of the fetal head

- features of early obstruction of labor (caput and moulding), and

- The fetal condition

In women with delay in the active phase of the first stage, every effort must be made to find a cause for the delay. This may either be due to inadequate contractions or obstruction due to CPD, malpresentation or malposition (such as occipito-posterior position), or a combination of these.

In cases of inadequate contractions:

- Amniotomy must be performed if membranes are still intact.

- Following that, the woman must be reassessed in two hours.

- In case there is inadequate progress, augmentation with oxytocin must be considered.

- The situation must be reassessed after four hours or earlier if required.

Multiparous women with delayed progress:

- Must be viewed with extreme caution.

- It is very important to exclude mechanical causes of delay before considering oxytocin.

- Use of oxytocin in multipara with obstructed labour could be extremely dangerous. 
In all cases where progress is slow in spite of adequate contractions a careful assessment must be made to exclude obstruction of labour.

Attention must be paid to effective pain relief and to correcting dehydration in these situations.

After paying attention to the above, cesarean section must be considered where the progress continues to be slow after four hours (less than two $\mathrm{cm}$ ) of commencing oxytocin.

\subsubsection{Management of second stage of labour}

\subsubsection{Passive second stage of labour (descent phase)}

- Is diagnosed when full cervical dilatation is reached in the absence of involuntary expulsive efforts by the mother.

- Bearing down must be discouraged at this stage.

- Intermittent auscultation of the fetal heart should be done immediately after a contraction for at least one minute, at least every 10 minutes. The maternal pulse should be palpated if there is suspected fetal bradycardia or any other FHR anomaly to differentiate the two heart rates.

- Presence of meconium must be noted.

\subsubsection{Active second stage of labour (expulsive phase)}

- Is diagnosed when the mother gets the urge to bear down with full dilatation.

- Intermittent auscultation of the fetal heart should be done immediately after a contraction for at least one minute, at least every 5 minutes. The maternal pulse should be palpated if there is fetal bradycardia or any other FHR anomaly.

- Presence of meconium must be noted.

Use of a hand-held Doppler device is recommended (in preference to a Pinnard stethoscope) for fetal heart rate monitoring in the second stage.

Women must be encouraged to continue consuming clear fluids during the second stage.

Support by the labour companion must be continued.

Total time durations allowed for the second stage of labour are as follows:

\section{Primigravida:}

- Birth would be expected to take place within 2 hours of the start of the active second stage in most women.

- A diagnosis of delay in the active second stage should be made when it has lasted 1 hour and need to seek the advice from a health professional trained in the assisted/operative vaginal birth if birth is not imminent.

\section{Multigravida:}

- Birth would be expected to take place within 1 hour of the start of the active second stage in most women.

- A diagnosis of delay in the active second stage should be made when it has lasted 30 minutes and requires advice from a health professional trained in assisted/ operative vaginal birth if birth is not imminent.

- Delay in the second stage in a multiparous woman must raise suspicion of disproportion or malposition.

One further hour is permitted for women in each category for women with epidural analgesia.

\subsubsection{Observations for women and babies in the second stage of labour:}

All observations should be documented on the partograph.

- Chart blood pressure and pulse hourly

- Continue four hourly temperature recording

- Vaginal examination must be offered after an hour in the active second stage after abdominal palpation and assessment of vaginal loss

- Half hourly documentation of frequency of contractions

- Consideration of the woman's emotional and psychological needs

In addition:

- Assessment of progress should include maternal behavior, effectiveness of pushing and fetal wellbeing, taking into account fetal position and station at the onset of the second stage. These factors will assist in deciding the timing of further vaginal examination and the need for obstetric review.

- Ongoing consideration should be given to the woman's position, hydration, coping strategies and pain relief throughout the second stage.

\subsubsection{Women's position and pushing in the second stage of labour:}

Although most deliveries in Sri Lanka are conducted in the dorsal/McRobert's position, women may be encouraged to adopt squatting, semi upright or lateral positions to aid the expulsion phase.

Women should be informed that in the second stage, they should be guided by their own urge to push.

If pushing is ineffective, strategies to assist birth such as support and encouragement and change of position can be used. 
In primigravida in whom contractions have become weak and there is no evidence of fetal compromise or obstruction, oxytocin may be administered as an infusion. In this case, the expulsive phase may be continued under close observation for a further 30 minutes. Delivery must be considered at the end of this period.

\subsubsection{Intrapartum interventions to reduce perineal trauma}

Either the 'hands on' (guarding the perineum and flexing the baby's head) or the 'hands poised' (with hands off the perineum and baby's head but in readiness) techniques can be used to facilitate spontaneous birth.

\section{A routine episiotomy should not be carried out during} spontaneous vaginal birth.

Episiotomy should only be performed selectively, in women in whom there is a clinical need such as instrumental birth or suspected fetal compromise or a high chance of perineal tears.

Where episiotomy is performed, mediolateral episiotomy, performed at 45 - 60 degrees from the midline directed to the right side, beginning at the vaginal fourchette is preferred to the median episiotomy. It should be performed at the time of crowning of the fetal head.

Episiotomy should be performed after infiltration of $1 \%$ lignocaine (up to $20 \mathrm{ml}$ may be used).

\subsubsection{Delivery}

The fetal head should not be allowed to extend till occiput is felt below the symphysis pubis. The perineum should be supported during delivery of the head. Once the head is delivered the woman should be discouraged from bearing down. Following restitution and external rotation, shoulders must be delivered with appropriately directed traction on the fetal head. The baby must be delivered onto the mother's abdomen. Breastfeeding should be initiated within 30 minutes of birth.

\subsubsection{Third stage of labour}

The third stage of labour is the period from complete delivery of the baby to the complete delivery of the placenta and membranes.

\subsubsection{Active management of the third stage of labour}

Active management of the third stage of labour is recommended for all mothers. This includes;

- Routine use of uetrotonic drugs: Oxytocin 5 IU intravenously soon after the delivery of the baby or $10 \mathrm{IU}$ intramuscularly

- Delayed cord clamping (2 minutes after the birth) and cutting of the cord
Delayed clamping of the cord allows for placental transfusion, which reduces neonatal and infant iron deficiency and anemia. This policy should be followed unless the baby is born in a poor condition or if the mother is bleeding or is Rhesus isoimmunized.

- Followed by controlled cord traction.

- This must be followed by uterine massage.

Clamp and cut the cord close to the perineum. A hand should be placed above the symphysis pubis to stabilize the uterus by applying counter traction during controlled cord traction. Application of cord traction when the uterus is relaxed could lead to acute inversion of the uterus.

After delivery, the placenta must be placed on a flat surface and the maternal surface examined for completeness. On the fetal surface the blood vessels must be traced to exclude a succenturiate lobe. Completeness of the fetal membranes must be ensured.

Observations in the immediate postpartum period include:

- Inspect for continued fresh bleeding

- Check pulse, blood pressure, uterine contraction and the level of the fundus every 15 minutes up to 2 hours

- Her general physical condition, as shown by her colour, respiration and her own report of how her feels

Experienced medical personnel should be informed in any one the following instances:

- Continuing fresh bleeding;

- Elevation of the level of the fundus;

- Increase of pulse rate above $\mathbf{1 0 0}$ or by $\mathbf{3 0}$ beats per minute;

- Drop in systolic blood pressure below $\mathbf{1 0 0}$ or by $30 \mathrm{mmHg}$.

The level of the fundus must be marked on the skin using a marker to make observations more objective.

\subsubsection{Delayed third stage}

Delayed third stage is diagnosed if the placenta is not delivered within 30 minutes of active management.

The first step in managing delayed third stage of labour is:

- To proceed to intraumbilical vein oxytocin, in a dose of $50 \mathrm{IU}$ in $30 \mathrm{ml}$ of $0.9 \%$ sodium chloride solution.

- A period of 30 minutes is allowed and controlled cord traction is attempted again. 
- If the placenta is not delivered by this method, manual removal of placenta is proceeded to.

\section{Care for the newborn baby}

Effective care at birth is needed for anticipation of problems with the transition from in utero dependent life to extra utero independent existence and to provide support to ensure stabilization.

- Skilled birth attendant (Medical Officers, Nursing Officers and Midwives) is responsible for the care.

- The care at birth is the same irrespective of birthing place or person attending to the birth.

- At least one health care provider trained in neonatal resuscitation must be physically available at time of birth of all infants irrespective of risk status.

- This person must be present in the delivery room before the birth of the baby.

- The attending personnel should document the baby details such as time of birth, weight, gender and any other relevant information in all cases.

The aims of neonatal care following birth include the following:

- Establishment of respiration (as per NRP guidelines)

- Prevention of hypothermia (Refer Newborn Guideline)

- Establishment of breast feeding (Refer Newborn Guideline)

- Prevention of infection (Refer Newborn Guideline)

- Detection of danger signs (Refer Newborn Guideline)

Following basic steps should be followed at the time of birth;

1. Call out the time of birth

2. Deliver the baby onto the mother's abdomen or into her arms

3. Dry baby with a warm towel or a warm piece of cloth

4. Wipe baby's eyes

5. Assess baby's breathing while drying

6. Make sure that there is no second baby

7. Change gloves or remove the first layer of gloves

8. Clamp and cut the umbilical cord

9. Put the baby between mother's breast for skin to skin care

10. Place the identity label on the baby

11. Cover mother and baby with warm cloth

12. Put a hat on baby's head
The Apgar score at 1 and 5 minutes should be recorded for all births.

Initiation of breastfeeding should be aimed for within 1 hour after birth.

Head circumference, birth weight, length and other measurement should be carried out once the first feed is complete. A health care professional should examine the baby to detect any physical abnormality and to identify any problems that require referral.

\section{Perineal care}

Perineal or genital trauma caused by either episotomy or tearing need to be repaired.

Before assessing for genital trauma:

- Explain to the woman what you are going to do and why

- Offer analgesia

- Ensure good lighting

- Position the woman so that she is comfortable and the genital structures can be seen clearly.

The initial assessment should be performed gently and with sensitivity and may be done in the immediate period following birth preferably as soon as the placenta is delivered.

\section{Classification of perineal trauma}

First degree: Injury to skin only

Second degree: Injury to the perineal muscles but not the anal sphincter

Third degree: Injury to the perineum involving the anal sphincter complex

Fourth degree: Injury to the perineum involving the anal sphincter complex and anal epithelium

Perineal repair should only be undertaken with tested effective analgesia in place using infiltration with up to $20 \mathrm{ml}$ of $1 \%$ lignocaine or equaling, or by topping up the epidural, as soon as possible by a medical officer.

The preferred suture material is rapidly absorbed polyglycolic acid.

The following basic principles should be observed when performing perineal repairs:

- Perineal trauma should be repaired using aseptic techniques.

- Equipment should be checked and swabs and needles counted before and after the procedure. 
- Good lighting is essential to see and identify the structures involved.

- Difficult injuries should be repaired by an experienced medical officer in theatre under regional or general anaesthesia. An indwelling catheter should be inserted for 24 hours to prevent urinary retention.

- Good anatomical alignment of the wound should be achieved, and consideration given to the cosmetic result.

- Rectal examination should be carried out after completing the repair to ensure that suture material has not accidently been inserted through the rectal mucosa.

- Following completion of repair, an accurate detailed account should be documented covering the extent of the trauma, the method of repair and the materials used.

- Information should be given to the woman regarding the extent of the trauma, pain relief, diet, hygiene and the importance of pelvic floor exercises. 